\title{
FAST Cl-TYPE INHIBITORY NEURON WITH DELAYED FEEDBACK HAS NON-MARKOV OUTPUT STATISTICS
}

\author{
A. K. Vidybida \\ Bogolyubov Institute for Theoretical Physics \\ 14-B, Metrologichna St., Kyiv, UA-03680, Ukraine \\ e-mail:vidybida@bitp.kiev.ua \\ (Received March 16, 2018)
}

\begin{abstract}
For a class of fast Cl-type inhibitory spiking neuron models with delayed feedback stimulated with a Poisson stochastic process of excitatory impulses, it is proven that the stream of output interspike intervals cannot be presented as a Markov process of any order.

Key words: Poisson stochastic process; spiking neuron; probability density function; delayed feedback; fast Cl-type inhibition; non-Markov stochastic process.
\end{abstract}

DOI: https://doi.org/10.30970/jps.22.4801

PACS number(s): 87.19.1l, 87.10.-e, 87.10.Ca, 87.10.Mn

\section{INTRODUCTION}

Spiking statistics of various neuronal models under a random stimulation has been studied in the framework of two main approaches. The first one is named in [1] as "Gaussian", because it describes random stimulation by means of Gaussian noise, see e.g. [2]. This approach has developed into the well-known diffusion approximation methodology, see [3]. The second approach is named in [1] as "quantal", because it takes into account the discrete nature of the influence any input impulse may have on its target neuron. The wide area of research and applications known as spiking neural networks, see [4] for a review, could be considered as utilizing the quantal approach. For a recent review of mathematically rigorous results regarding neuronal the spiking statistics in the both approaches see [5]. We study here mathematically rigorously, in the framework of quantal approach, spiking statistics of the inhibitory neuron model belonging to a class of models (see Sec. II, below) with fast Cl-type inhibitory delayed feedback (see Fig. 1, below).

\section{A. Biological inspiration}

The neurons which send inhibitory impulses onto their own body or dendrites are known in real nervous systems, see [20-23]. The chief inhibitory neurotransmitter in the nervous system is Gamma-aminobutyric acid (GABA). The GABA can activate several types of receptors, the main of which are $\mathrm{GABA}_{a}$ and $\mathrm{GABA}_{b}$. If $\mathrm{GABA}_{a}$ receptors are activated, the excitable membrane becomes permeable for $\mathrm{Cl}^{-}$ions. If a neuron is partially excited, that is its membrane is depolarized, the $\mathrm{Cl}^{-}$current cancels this depolarization since the $\mathrm{Cl}^{-}$reversal potential is close/equal to the resting potential. For the same reason, the $\mathrm{Cl}^{-}$current through open $\mathrm{GABA}_{a}$ channels does not appear, if the membrane is at its resting potential.

A different case is $\mathrm{GABA}_{b}$ receptors activation. This causes $\mathrm{K}^{+}$ions permeability. The outward $\mathrm{K}^{+}$current is able to hyperpolarize the membrane even below its resting potential.

The remarkable difference between $\mathrm{GABA}_{a}$ and $\mathrm{GABA}_{b}$ mediated inhibition is rather different kinetics of the corresponding $\mathrm{Cl}^{-}$and $\mathrm{K}^{+}$currents. Namely, according to [24], the $\mathrm{Cl}^{-}$current rise time is $1-5 \mathrm{~ms}$, and the decay time constant is about $10-25 \mathrm{~ms}$. The $\mathrm{K}^{+}$current rise time is $10-120 \mathrm{~ms}$, and the decay time constant is about $200-1600 \mathrm{~ms}$. The $\mathrm{K}^{+}$current can be even slower, see $[22,25,26]$.

Inspired by this contrast in the speed of $\mathrm{Cl}^{-}$and $\mathrm{K}^{+}$ transients, we idealize the $\mathrm{Cl}^{-}$current kinetics as having infinitesimally short rise time and infinitely fast decay, both can be achieved with infinitely large $\mathrm{Cl}^{-}$conductance at the moment of receiving inhibitory impulse. This kind of the $\mathrm{Cl}^{-}$current kinetics does ensure the perfect reset of the membrane voltage to the resting state at the moment when an inhibitory impulse arrives. Within the limited experimental data set available for inhibitory autapses, see [24], a single impulse delivered through a single synapse ensures only a partial reset. At this point, our statement of the problem diverges from the current data. At the same time, in the artificial neuromorphic systems, see [6,7], a complete reset may well be realized. Considering a partial reset would inappropriately increase the paper's dimensions.

In the following, it is assumed that a neuron sends back to itself its output impulses through a delayed feed-

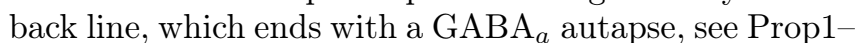
Prop3, in Sec. II B. This construction is stimulated with a Poisson stream of excitatory input impulses. For this configuration it has been proven in the previous paper [8] for the case of a concrete neuronal model - the inhibito-

\footnotetext{
${ }^{1}$ Detailed description of the binding neuron model can be found in [9]. See also https://en.wikipedia.org/wiki/Binding_neuron.
} 


\section{A. K. VIDYBIDA}

ry binding neuron ${ }^{1}$ with threshold 2 - that statistics of its interspike intervals (ISIs) is essentially non-Markov². In paper [13], it has been proven for the Poisson input and for a class of excitatory neuronal models that the presence of delayed feedback makes their activity nonMarkov. In this paper, we use the approach developed in [13] in order to refine and extend the methods of [8] making them applicable to any inhibitory neuron with fast Cl-type inhibition satisfying a number of simple and natural conditions, see Cond0-Cond4, below. The stimulation is assumed to be a Poisson stochastic process. Under those conditions, we prove rigorously that ISI statistics of a neuron with delayed fast Cl-type inhibitory feedback stimulated with a Poisson point stochastic process of input impulses cannot be represented as a Markov chain of any finite order. Finally, it should be mentioned that our consideration is valid also for artificial hardware neurons, see $[27,28]$, and abstract neurons used in mathematical studies, provided that Cond0-Cond4 and Prop1-Prop3, below, are satisfied.

\section{DEFINITIONS AND ASSUMPTIONS}

\section{A. Neuron without feedback}

We assume that a neuron satisfies the following conditions:

- Cond0: Neuron is deterministic: Identical stimuli elicit identical spike trains from the same neuron.

- Cond1: Neuron is stimulated with a Poisson input stationary stream of excitatory impulses.

- Cond2: Neuron may fire a spike only at the moment when it receives an input impulse.

- Cond3: Immediately after firing, neuron appears in its resting state.

- Cond4: The output interspike interval (ISI) distribution can be characterized with a probability density function (pdf) $p^{0}(t)$, which is continuous with

$$
p^{0}(0)=0,
$$

positive:

$$
t>0 \Rightarrow p^{0}(t)>0
$$

and bounded:

$$
\sup _{t>0} p^{0}(t)<\infty
$$

By $t$ we denote the ISI's length. Also, we impose on the function $p^{0}(t)$ the following condition: $t<0 \Rightarrow p^{0}(t)=0$ in order to have it defined for all real numbers.

These conditions are, with some modifications, similar to those assumed in [13] for a class of excitatory neurons. The modifications are as follows:

Cond3 - we assume that after firing a neuron appears in its resting state with all excitation canceled, while in [13] it is a standard state, which not necessarily is the resting one.

Cond 4 - the requirement of continuity of $p^{0}(t)$ is added as compared to [13]. This addition has a pure mathematical nature and seems to be valid for any "good" neuronal model (without feedback). The subsequent proof of non-Markovianity relies on it.

The Cond3 above, limits the set of models as compared to [13]. Namely, it claims that the standard state of $[13$, Cond3 $]$ must be exactly the resting state of a neuron. This requirement is imposed due to the specifics of Cl-type fast inhibition. For our approach, it is important that after receiving an inhibitory impulse, the neuron appears in exactly the same state as immediately after firing. And the state after receiving thetype inhibitory impulse can be only the resting state, see Sec. I A, above. It seems that these conditions are satisfied for many threshold-type neuronal models known in the literature, see [14-17] and citations therein. But this still has to be proven by calculating corresponding $p^{0}(t)$. At least, all the five conditions are satisfied for the binding neuron model and for the basic leaky integrate-and-fire (LIF) model, both for the Poisson stimulation. See $[18,19]$, where $p^{0}(t)$ is calculated exactly for any of the two models mentioned, respectively.

\section{B. Feedback line action}

We expect that the feedback line satisfies Prop1, Prop2 of [13], which are reproduced below for completeness. The Prop3 of [13] should be modified for the Cl-type fast inhibition as shown below:

- Prop1: The time delay in the line is $\Delta>0$.

- Prop2: The line is able to convey no more than one impulse.

\footnotetext{
${ }^{2}$ Sometimes, a concept of a point stochastic Markov process is confused with a process whose consecutive realizations are uncorrelated. Actually, the latter is a renewal process, which is a specific case of Markov process. As regards a general Markov process, its realizations can well be correlated, see e.g. [10]. In this paper, we do not study the correlations (which itself is an interesting topic, which could be addressed separately), but prove that the output statistics does not have the Markov property as it is defined, e.g., in [11, Ch.2 §6]. Interesting remarks about the physics of non-Markovianity can be found in [12].
} 
- Prop3: The impulse conveyed to the neuronal input is the fast Cl-type inhibitory impulse. This means that after receiving such an impulse, the neuron appears in its resting state. This is the only action of the inhibitory impulse in the sense that it has no influence on further neuronal states created by next excitatory impulses. It does not affect the neuron being in its resting state either.

Prop1 expects that the delay is always the same and each impulse entering the line is delivered to its output and effects the neuron. Thus, we do not consider here cases when the transmission is unreliable, or the delay time is not constant.

The validity of Prop2 depends on the relation between the conduction velocity, recovery time and the line's length. Also Prop2 seems plausible if the firing frequency is low.

Prop3 just characterizes a neuronal model as inhibitory with $\mathrm{GABA}_{a}$-type autapse. Its validity depends on the fact that the $\mathrm{Cl}^{-}$reversal potential is identical to the resting potential. In some cases this is fulfilled, see [24]. It is also expected that a single action potential delivered by a feedback line is potent enough for canceling any excitation present. Taking into account that the observed single $\mathrm{GABA}_{a}$ IPSP peak value rare exceeds $6 \mathrm{mV}$, this may require the delay line sprouting into several autaptic endings.

The consequence of Prop2 above, important for us is that at any moment of time the feedback line is either empty, or conveys a single impulse. If it does convey an impulse, then its state can be described with a stochastic variable $s, s \in] 0 ; \Delta]$, which further we call "time to live", see Fig. 1. The variable $s$ denotes the exact time required by the impulse to reach the output end of the line, which is the neuron's input for inhibitory impulses, and to leave the line with the consequences described in Prop3, above. Here, $\Delta$ denotes the delay duration in the feedback line. Notice, that at the beginning of any ISI, the line is never empty.

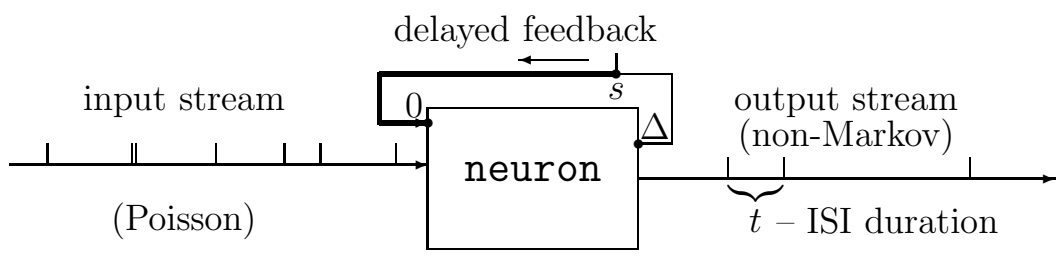

Fig. 1. Neuron with delayed feedback. Neuron in the figure, stands for any neuronal model, which satisfies the set of conditions Cond0-Cond4, above.

\section{RESULTS}

Let $p^{\text {inh }}\left(t_{n+1} \mid t_{n}, \ldots, t_{0}\right) d t_{n+1}$ denote the conditional probability to get the duration of $(n+2)$-nd ISI in the interval $\left[t_{n+1} ; t_{n+1}+d t_{n+1}\right.$ [ provided that previous $n+1$ ISIs have duration $t_{n}, \ldots, t_{0}$, respectively. From the definition in [11, Ch.2 I6], one can obtain the necessary condition

$$
\begin{aligned}
& p^{\mathrm{inh}}\left(t_{n+1} \mid t_{n}, \ldots, t_{1}, t_{0}\right) \\
& =p^{\mathrm{inh}}\left(t_{n+1} \mid t_{n}, \ldots, t_{1}\right),
\end{aligned}
$$

required for the stochastic process $\left\{t_{j}\right\}$ to be $n$th order Markov chain. Notice, that (4) must be satisfied for any values of the variables $t_{i}, i=0, \ldots, n+1$.

Our purpose here is to prove the following Theorem ${ }^{3}$ :

Theorem 1. A neuronal model satisfies conditions Cond0-Cond4, above. Suppose that the model is extended by introducing a delayed fast Cl-type inhibitory feedback line, which satisfies the Prop1-Prop3, above. Then, in the stationary regime, the output stream of ISIs of the neuron cannot be presented as a Markov chain of any finite order.

\section{A. Proof outline}

We intend to prove that relation (4) does not hold for any $n$. For this purpose, we calculate exact expression for $p^{\text {inh }}\left(t_{n+1} \mid t_{n}, \ldots, t_{0}\right)$ as

$$
\begin{aligned}
& p^{\mathrm{inh}}\left(t_{n+1} \mid t_{n}, \ldots, t_{0}\right. \\
& =\frac{p^{\mathrm{inh}}\left(t_{n+1}, t_{n}, \ldots, t_{0}\right)}{p^{\mathrm{inh}}\left(t_{n}, \ldots, t_{0}\right)}
\end{aligned}
$$

from which it will be clearly seen that the $t_{0}$-dependence in $p^{\text {inh }}\left(t_{n+1} \mid t_{n}, \ldots, t_{0}\right)$ cannot be eliminated whatever large the $n$ is. In Eq. (5), expression $p^{\mathrm{inh}}\left(t_{n}, \ldots, t_{0}\right)$ denotes the joint probability density function to have $n+1$ consecutive ISIs $\left\{t_{n}, \ldots, t_{0}\right\}$ in a neuron with the fast Cl-type inhibitory delayed feedback.

Let us introduce the conditional joint probability density $p^{\mathrm{inh}}\left(t_{n+1}, \ldots, t_{0} \mid s\right)$, which denotes the conditional probability density to get $n+2$ consecutive ISIs $\left\{t_{n+1}, \ldots, t_{0}\right\}$ provided that at the beginning of the first

\footnotetext{
${ }^{3} \mathrm{~A}$ similar theorem for the excitatory feedback line has been proven in [13].
} 


\section{A. K. VIDYBIDA}

ISI $\left(t_{0}\right)$ the time to live of the impulse in the feedback line is equal to $s$. This conditional probability can be used to calculate required joint pdfs as follows

$$
\begin{aligned}
& p^{\mathrm{inh}}\left(t_{n+1}, \ldots, t_{0}\right) \\
& =\int_{0}^{\Delta} p^{\mathrm{inh}}\left(t_{n+1}, \ldots, t_{0} \mid s\right) f^{\mathrm{inh}}(s) d s,
\end{aligned}
$$

where $f^{\text {inh }}(s)$ is the stationary pdf which describes the distribution of times to live at the beginning of any ISI in the stationary regime.

In what follows, we analyze the structure of functions $f^{\text {inh }}(s)$ and $p^{\text {inh }}\left(t_{n+1}, \ldots, t_{0} \mid s\right)$. It appears that $f^{\text {inh }}(s)$ has a singular component $a \delta(s-\Delta)$ with $a>0$, and $p^{\text {inh }}\left(t_{n+1}, \ldots, t_{0} \mid s\right)$ has jump discontinuities at definite hyper-planes in the $(n+3)$-dimensional space of its variables $\left(t_{n+1}, \ldots, t_{0}, s\right)$. After integration in (6), some of those discontinuities will survive in the $(n+2)$ dimensional space of variables $\left(t_{n+1}, \ldots, t_{0}\right)$, and exactly one of those that survived has its position depending on $t_{0}$. The $t_{0}$-dependent jump discontinuity will also survive in the $p^{\mathrm{inh}}\left(t_{n+1} \mid t_{n}, \ldots, t_{0}\right)$ for any $n$, provided that $t_{n}, \ldots, t_{0}$ satisfy the following condition:

$$
\sum_{i=0}^{n} t_{i}<\Delta,
$$

where $\Delta>0$ is the full delay time in the feedback line. Taking into account that the equation in the necessary condition (4) must hold for any set of $t_{n+1}, \ldots, t_{0}$, we conclude that (4) cannot be satisfied for any $n$.

\section{B. The proof}

1. Structure of functions $p^{\mathrm{inh}}\left(t_{n+1}, \ldots, t_{0} \mid s\right)$

The specifics of the feedback line action together with condition (7) results in a very simple structure of $p^{\mathrm{inh}}\left(t_{n+1}, \ldots, t_{0} \mid s\right)$ at different parts of the integration domain in (6). Those parts are defined as follows:

$$
\begin{aligned}
& D_{k}=\left\{s \mid \sum_{i=0}^{k-1} t_{i}<s \leq \sum_{i=0}^{k} t_{i}\right\}, \\
& k=0, \ldots, n, \\
& D_{n+1}=\left\{s \mid \sum_{i=0}^{n} t_{i}<s \leq \Delta\right\}, .
\end{aligned}
$$

As regards the structure itself, the following representation can be derived similarly as it was done in [13]:

$$
\begin{aligned}
& p^{\text {inh }}\left(t_{n+1}, \ldots, t_{0} \mid s\right) \\
& =p^{\text {inh }}\left(t_{n+1}, \ldots, t_{k+1} \mid \Delta\right) \\
& \times p^{\text {inh }}\left(t_{k} \mid s-\sum_{i=0}^{k-1} t_{i}\right) \prod_{i=0}^{k-1} p^{0}\left(t_{i}\right), \\
& s \in D_{k}, \quad k=0, \ldots, n,
\end{aligned}
$$

$$
\begin{aligned}
& p^{\text {inh }}\left(t_{n+1}, \ldots, t_{0} \mid s\right) \\
& =p^{\text {inh }}\left(t_{n+1} \mid s-\sum_{i=0}^{n} t_{i}\right) \prod_{i=0}^{n} p^{0}\left(t_{i}\right), \\
& s \in D_{n+1} .
\end{aligned}
$$

$$
\begin{aligned}
& p^{\operatorname{inh}}\left(t_{n+1}, \ldots, t_{k+1} \mid \Delta\right) \\
& p^{\operatorname{inh}}\left(t_{n+1} \mid \Delta-\sum_{i=k+1}^{n} t_{i}\right) \prod_{i=k+1}^{n} p^{0}\left(t_{i}\right) .
\end{aligned}
$$

Here $p^{\text {inh }}(t \mid s)$ denotes the conditional pdf to get ISI of duration $t$ if at its beginning, the time to live of the impulse in the feedback line is $s$.

The representation of $p^{\text {inh }}\left(t_{n+1}, \ldots, t_{0} \mid s\right)$ by means of $p^{0}(t)$ and $p^{\mathrm{inh}}(t \mid s)$ found here is similar to that found in [13] for the excitatory case. But the structure of function $p^{\text {inh }}(t \mid s)$, used in that representation, is different.

\section{Structure of function $p^{\mathrm{inh}}(t \mid s)$}

Expect that at the beginning of an ISI, there is an impulse in the feedback line with time to live $s$. Then the probability that this ISI will have its duration $t<s$ does not depend on the feedback line presence. Therefore,

$$
t<s \Rightarrow p^{\mathrm{inh}}(t \mid s)=p^{0}(t) .
$$

In the opposite situation, receiving of an ISI duration greater than $s$ happens if (i) the neuron is silent during interval $] 0 ; s[$ and (ii) the neuron starts at its resting state (Prop3, above) at the moment $s$ and fires at $t>s$. The realizations of events (i) and (ii) depend on disjoint segments of the Poisson input stream (Cond1, above). Therefore, (i) and (ii) are statistically independent. The probability of (i) is as follows:

$$
\mathbf{P}^{0}(s)=1-\int_{0}^{s} p^{0}(t) d t .
$$

The probability of (ii) is $p^{0}(t-s)$. This gives for $t>s$

$$
p^{\mathrm{inh}}(t \mid s)=\mathbf{P}^{0}(s) p^{0}(t-s) .
$$

It can be concluded from (11) and (13) that

$$
\lim _{t \uparrow s} p^{\mathrm{inh}}(t \mid s)=p^{0}(s), \lim _{t \downarrow s} p^{\mathrm{inh}}(t \mid s)=0 .
$$

Now, taking into account (1) and (2) from Cond4, above, we conclude that the function $p^{\mathrm{inh}}(t \mid s)$ considered as a function of two variables $(t, s), t \geq 0, s \in] 0 ; \Delta]$ has a jump discontinuity along the straight line $t=s$. The magnitude of this jump is $p^{0}(s)$, and it is strictly positive 
for positive $t$. Concrete values of $p^{\text {inh }}(t \mid s)$ along the line $t=s$ do not matter and can be chosen arbitrarily.

Finally, for $p^{\text {inh }}(t \mid s)$ we have ${ }^{4}$

$$
p^{\mathrm{inh}}(t \mid s)=\chi(s-t) p^{0}(t)+\mathbf{P}^{0}(s) p^{0}(t-s),
$$

where $\chi(s)$ is the Heaviside step function.

\section{Structure of probability density function $f^{\mathrm{inh}}(s)$}

Everywhere in this paper we expect that all pdfs $p^{\text {inh }}\left(t_{n+1}, \ldots, t_{0}\right)$ have achieved their stationary form, and we analyze the stationary regime. But any stationary regime arises from some initial distribution. In principle, different initial distributions may result in different final stationary distributions.

As can be concluded from (8)-(10), the only quantity, which might depend on the initial conditions in the right-hand side of representation (6) is the pdf $f^{\mathrm{inh}}(s)$.

Before the stationary regime is achieved, $f^{\text {inh }}(s)$ is changed after each firing:

$$
f_{n+1}(s)=\int_{0}^{\Delta} \mathbf{P}\left(s \mid s^{\prime}\right) f_{n}\left(s^{\prime}\right) d s^{\prime},
$$

where the transition function $\mathbf{P}\left(s \mid s^{\prime}\right)$ gives the probability density to find at the beginning of an ISI an impulse in the line with time to live $s$ provided that at the beginning of the previous ISI, there was an impulse with time to live $s^{\prime}$. In the stationary regime, the pdf $f(s)$ must satisfy the following equation

$$
f^{\mathrm{inh}}(s)=\int_{0}^{\Delta} \mathbf{P}\left(s \mid s^{\prime}\right) f^{\mathrm{inh}}\left(s^{\prime}\right) d s^{\prime},
$$

Now, the question of existence and uniqueness of the stationary regime might be resolved by analyzing Eqs. (15) and (16) for convergence and uniqueness. This is expected to be done in another paper. In this paper we assume that the sequence $\left\{f_{n}^{\text {inh }}(s)\right\}$ of pdfs generated by Eq. (15) converges to some pdf for any initial $f_{0}(s)$, and admit that there might be different limiting distributions for different $f_{0}(s)$. The exact expression for $\mathbf{P}\left(s \mid s^{\prime}\right)$ is found in [13, Eqs.(11)-(13)]. It appears that the structure of $f^{\text {inh }}(s)$, which follows from (16) is exactly the same as has been found in [13] for the excitatory case. This structure is as follows ${ }^{5}$

$$
f^{\mathrm{inh}}(s)=g(s)+a \delta(s-\Delta),
$$

where $a>0$ and $g(s)$ is a bounded continuous function vanishing out of interval $] 0 ; \Delta[$.

$$
\begin{aligned}
& \text { 4. Forms of } p^{\mathrm{inh}}\left(t_{n+1}, \ldots, t_{0}\right) \text { and } p^{\mathrm{inh}}\left(t_{n}, \ldots, t_{0}\right) \text { after } \\
& \text { integration in }(6)
\end{aligned}
$$

Let $D=\bigcup_{k=0}^{n} D_{k}$. At $D$, representations (8) and (10) are valid. Also at $D, f^{\mathrm{inh}}(s)$ reduces to $g(s)$. Therefore,

$$
\begin{aligned}
& \int_{D} p^{\mathrm{inh}}\left(t_{n+1}, \ldots, t_{0} \mid s\right) f^{\mathrm{inh}}(s) d s \\
& =\sum_{k=0}^{n} p^{\mathrm{inh}}\left(t_{n+1} \mid \Delta-\sum_{i=k+1}^{n} t_{i}\right) \\
& \times \prod_{\substack{i=0 \\
i \neq k}}^{n} p^{0}\left(t_{i}\right) \int_{D_{k}} p^{\mathrm{inh}}\left(t_{k} \mid s-\sum_{j=0}^{k-1} t_{j}\right) g(s) d s .
\end{aligned}
$$

The first factor (with fixed $k, 0 \leq k \leq n$ ) in the r.h.s. of Eq. (18) is as follows:

$$
p^{\text {inh }}\left(t_{n+1} \mid \Delta-\sum_{i=k+1}^{n} t_{i}\right) .
$$

Due to Eq. (14), this factor does have a jump discontinuity along the hyperplane $\sum_{i=k+1}^{n+1} t_{i}=\Delta$ in the space of variables $\left(t_{0}, \ldots, t_{n+1}\right)$. Notice, that the position of this hyperplane does not depend on $t_{0}$ for any $k \in\{0, \ldots, n\}$.

The second factor in the r.h.s. of Eq. (18) is as follows: $\prod_{i=0}^{n} p^{0}\left(t_{i}\right)$, and it is continuous. $i \neq k$

The third factor in the r.h.s. of Eq. (18) can be transformed as follows:

$$
\begin{aligned}
& \int_{D_{k}} p^{\text {inh }}\left(t_{k} \mid s-\sum_{j=0}^{k-1} t_{j}\right) g(s) d s \\
= & \int_{j=0}^{\sum_{j=0}^{k} t_{j}} p^{\text {inh }}\left(t_{k} \mid s-\sum_{j=0}^{k-1} t_{j}\right) g(s) d s \\
& \sum_{j=0}^{t_{k}} t_{j} p^{\text {inh }}\left(t_{k} \mid s\right) g\left(s+\sum_{j=0}^{k-1} t_{j}\right) d s \\
= & \int_{0}^{t_{k}} \mathbf{P}^{0}(s) p^{0}\left(t_{k}-s\right) g\left(s+\sum_{j=0}^{k-1} t_{j}\right) d s .
\end{aligned}
$$

\footnotetext{
${ }^{4}$ Compare this with [29, Eq. (11)], where $p^{\text {inh }}(t \mid s)$ is calculated exactly for the binding neuron model stimulated with a Poisson stream.

${ }^{5}$ Compare this with [30, Eqs. (14)-(16)], where $f(s)$ is calculated exactly for the binding neuron model.
} 


\section{A. K. VIDYBIDA}

The last expression is continuous with respect to variables $\left(t_{0}, \ldots, t_{n+1}\right)$. Therefore, one can conclude that expression (18) does not have a jump discontinuity whose position depends on $t_{0}$.

Consider now the remaining part of the integral in (6). With (9) taken into account one has:

$$
\begin{aligned}
& \int_{D_{n+1}} p^{\mathrm{inh}}\left(t_{n+1}, \ldots, t_{0} \mid s\right) f^{\mathrm{inh}}(s) d s \\
= & \prod_{i=0}^{n} p^{0}\left(t_{i}\right) \int_{D_{n+1}} p^{\mathrm{inh}}\left(t_{n+1} \mid s-\sum_{i=0}^{n} t_{i}\right) f^{\mathrm{inh}}(s) d s .
\end{aligned}
$$

Here, the first factor, $\prod_{i=0}^{n} p^{0}\left(t_{i}\right)$ is continuous and strictly positive for positive $t_{i}$. The second factor can be transformed as follows:

$$
\begin{aligned}
& \int_{D_{n+1}} p^{\mathrm{inh}}\left(t_{n+1} \mid s-\sum_{i=0}^{n} t_{i}\right) f^{\mathrm{inh}}(s) d s \\
= & \int_{\sum_{i=0}^{n} t_{i}}^{\Delta} p^{\mathrm{inh}}\left(t_{n+1} \mid s-\sum_{i=0}^{n} t_{i}\right) f^{\mathrm{inh}}(s) d s \\
= & \int_{0}^{\Delta-\sum_{i=0}^{n} t_{i}} p^{\mathrm{inh}}\left(t_{n+1} \mid s\right) f^{\mathrm{inh}}\left(s+\sum_{i=0}^{n} t_{i}\right) d s .
\end{aligned}
$$

Now, let us use representations (14) and (17) in order to figure out which kind of discontinuities expression (21) has. Due to (14) and (17), expression (21) will have four terms. The first one is obtained by choosing the first term both in (14) and (17):

$$
A_{11}=\int_{0}^{\Delta-\sum_{i=0}^{n} t_{i}} \chi\left(s-t_{n+1}\right) p^{0}\left(t_{n+1}\right) g\left(s+\sum_{i=0}^{n} t_{i}\right) d s
$$

This term is either equal to zero, if $t_{n+1}>\Delta-\sum_{i=0}^{n} t_{i}$, or otherwise transforms into a continuous function of variables $\left(t_{0}, \ldots, t_{n+1}\right)$. Moreover,

$$
\lim _{t_{n+1} \uparrow \Delta-\sum_{i=0}^{n} t_{i}} A_{11}\left(t_{n+1}\right)=0 .
$$

The second one is obtained by choosing the second term in (14) and the first term in (17):

$$
A_{21}=\int_{0}^{\Delta-\sum_{i=0}^{n} t_{i}} \mathbf{P}^{0}(s) p^{0}\left(t_{n+1}-s\right) g\left(s+\sum_{i=0}^{n} t_{i}\right) d s .
$$

This is also a continuous function of variables $\left(t_{0}, \ldots, t_{n+1}\right)$.

The third one is obtained by choosing the first term in (14) and the second term in (17):

$$
\begin{aligned}
& A_{12}=a \int_{0}^{\Delta-\sum_{i=0}^{n} t_{i}} \chi\left(s-t_{n+1}\right) p^{0}\left(t_{n+1}\right) \\
& \times \delta\left(\sum_{i=0}^{n} t_{i}+s-\Delta\right) d s=a \chi\left(\Delta-\sum_{i=0}^{n+1} t_{i}\right) p^{0}\left(t_{n+1}\right) .
\end{aligned}
$$

This term has a jump discontinuity along the hyperplane

$$
\sum_{i=0}^{n+1} t_{i}=\Delta
$$

The forth one is obtained by choosing the second term in (14) and the second term in (17):

$$
\begin{aligned}
A_{22}= & a \int_{0}^{\Delta-\sum_{i=0}^{n} t_{i}} \mathbf{P}^{0}(s) p^{0}\left(t_{n+1}-s\right) \\
& \times \delta\left(\sum_{i=0}^{n} t_{i}+s-\Delta\right) d s= \\
= & \mathbf{P}^{0}\left(\Delta-\sum_{i=0}^{n} t_{i}\right) p^{0}\left(\sum_{i=0}^{n+1} t_{i}-\Delta\right) .
\end{aligned}
$$

This is also a continuous function of variables $\left(t_{0}, \ldots, t_{n+1}\right)$.

After taking into account the above reasoning, we conclude that the required joint probability density has the following form

$$
\begin{aligned}
& p^{\mathrm{inh}}\left(t_{n+1}, \ldots, t_{0}\right)=p^{w}\left(t_{n+1}, \ldots, t_{0}\right) \\
& +a \chi\left(\Delta-\sum_{i=0}^{n+1} t_{i}\right) \prod_{j=0}^{n+1} p^{0}\left(t_{j}\right),
\end{aligned}
$$

where function $p^{w}\left(t_{n+1}, \ldots, t_{0}\right)$ does not have a jump discontinuity depending on $t_{0}$, and the second term in (24) does have such a discontinuity along the hyperplane (23).

Form of $p^{\mathrm{inh}}\left(t_{n}, \ldots, t_{0}\right)$ after integration

Assuming that (7) is satisfied we have similarly to (8), (9)

$$
\begin{aligned}
& p^{\mathrm{inh}}\left(t_{n}, \ldots, t_{0} \mid s\right)=p^{\mathrm{inh}}\left(t_{n}, \ldots, t_{k+1} \mid \Delta\right) \\
& \times p^{\mathrm{inh}}\left(t_{k} \mid s-\sum_{i=0}^{k-1} t_{i}\right) \prod_{i=0}^{k-1} p^{0}\left(t_{i}\right), \\
& s \in D_{k}, \quad k=0, \ldots, n-1,
\end{aligned}
$$




$$
\begin{aligned}
& p^{\mathrm{inh}}\left(t_{n}, \ldots, t_{0} \mid s\right)=p^{\mathrm{inh}}\left(t_{n} \mid s-\sum_{i=0}^{n-1} t_{i}\right) \prod_{i=0}^{n-1} p^{0}\left(t_{i}\right), \\
& s \in D_{n} .
\end{aligned}
$$

Again due to (7), and in analogy with (10) instead of the last two equations we have the following one:

$$
\begin{aligned}
& p^{\mathrm{inh}}\left(t_{n}, \ldots, t_{0} \mid s\right)=p^{\mathrm{inh}}\left(t_{k} \mid s-\sum_{i=0}^{k-1} t_{i}\right) \prod_{\substack{i=0 \\
i \neq k}}^{n} p^{0}\left(t_{i}\right) \\
& s \in D_{k}, k=0, \ldots, n .
\end{aligned}
$$

It is clear that the expression similar to (9) turns here into the following

$$
p^{\mathrm{inh}}\left(t_{n}, \ldots, t_{0} \mid s\right)=\prod_{i=0}^{n} p^{0}\left(t_{i}\right), s \in D_{n+1} .
$$

Now, due to (25), (26) we have

$$
\begin{aligned}
& p^{\mathrm{inh}}\left(t_{n}, \ldots, t_{0}\right)=\int_{0}^{\Delta} p^{\mathrm{inh}}\left(t_{n}, \ldots, t_{0} \mid s\right) f^{\mathrm{inh}}(s) d s \\
& =\sum_{\substack{k=0 \\
i \neq 0 \\
i \neq k}}^{n} p^{0}\left(t_{i}\right) \int_{D_{k}} p^{\mathrm{inh}}\left(t_{k} \mid s-\sum_{i=0}^{k-1} t_{i}\right) g(s) d s \\
& +\prod_{i=0}^{n} p^{0}\left(t_{i}\right) \int_{D_{n+1}} f^{\mathrm{inh}}(s) d s .
\end{aligned}
$$

From calculations similar to those made in Eq. (19) it can be concluded that $p^{\operatorname{inh}}\left(t_{n}, \ldots, t_{0}\right)$ is continuous at the domain defined by $(7)$.

$$
\begin{aligned}
& \text { 5. } t_{0} \text {-dependence cannot be eliminated in } \\
& p^{\text {inh }}\left(t_{n+1} \mid t_{n}, \ldots, t_{0}\right)
\end{aligned}
$$

Now, with representations $(24)$ for $p^{\text {inh }}\left(t_{n+1}, \ldots, t_{0}\right)$ and (27) for $p^{\text {inh }}\left(t_{n}, \ldots, t_{0}\right)$ we can pose a question about the form of $p^{\mathrm{inh}}\left(t_{n+1} \mid t_{n}, \ldots, t_{0}\right)$. The latter can be found as defined in (5). First of all, notice that due to (27) and Cond $4, p^{\text {inh }}\left(t_{n}, \ldots, t_{0}\right)$ is strictly positive for positive ISIs. This allows us to use it as denominator in definition (5). Second, it can be further concluded from $(27)$ and Cond 4 that $p^{\text {inh }}\left(t_{n}, \ldots, t_{0}\right)$ is bounded. The latter together with the continuity of $p^{\text {inh }}\left(t_{n}, \ldots, t_{0}\right)$ means that any discontinuity of jump type present in $p^{\text {inh }}\left(t_{n+1}, \ldots, t_{0}\right)$ appears also in $p^{\text {inh }}\left(t_{n+1} \mid t_{n}, \ldots, t_{0}\right)$. It follows from the above and from the Eq. (24) that the conditional pdf $p^{\text {inh }}\left(t_{n+1} \mid t_{n}, \ldots, t_{0}\right)$ can be represented in the following form:

$$
\begin{aligned}
& p^{\mathrm{inh}}\left(t_{n+1} \mid t_{n}, \ldots, t_{0}\right) \\
& =p^{w}\left(t_{n+1} \mid t_{n}, \ldots, t_{0}\right) \\
& +Z\left(t_{n+1}, \ldots, t_{0}\right) \chi\left(\Delta-\sum_{i=0}^{n+1} t_{i}\right),
\end{aligned}
$$

where $p^{w}\left(t_{n+1} \mid t_{n}, \ldots, t_{0}\right)$ does not have any jump type discontinuity whose position depends on $t_{0}$, and $Z\left(t_{n+1}, \ldots, t_{0}\right)$ is a strictly positive function:

$$
Z\left(t_{n+1}, \ldots, t_{0}\right)=\frac{a \prod_{i=0}^{n+1} p^{0}\left(t_{i}\right)}{p\left(t_{n}, \ldots, t_{0}\right)} .
$$

The second summand in (28) thus has a jump discontinuity, whose position depends on $t_{0}$ and this discontinuity cannot be counterbalanced by the terms accumulated in the first summand. Therefore, representation (28) proves that for any $n$, conditional pdf $p^{\text {inh }}\left(t_{n+1} \mid t_{n}, \ldots, t_{0}\right)$ does depend on $t_{0}$ (the second term in (28)) and this dependence cannot be eliminated.

See also Appendix below, where the above general reasoning is illustrated for the LIF neuronal model with threshold 2 (that is two input impulses applied in a short succession are able to trigger, see (31), below).

\section{DISCUSSION AND CONCLUSIONS}

The question as to what extent the stream of neuronal output impulses can be modeled as a Poisson stream has been discussed in neurophysics, see [31]. The experimentally observed presence of memory in the ISIs output of real neurons has been reported many times, see [32-36]. Also several theoretical models of how the memory could appear are offered, see [15,37-41,43].

In this paper, we use the quantal approach as it is defined in [1] in order to prove that the Markov property is broken in the ISI output stream of a neuronal model belonging to a defined class of models equipped with delayed fast Cl-type inhibitory feedback, which is stimulated with a Poisson stochastic process of excitatory input impulses. The Theorem proven (see page 3 ) claims that in a normal biophysical construction, one may not expect even a renewal, let alone a Poisson type of activity. Several previous results obtained in the quantal approach are used in this paper. The first results this paper is grounded on are obtained for the binding neuron (BN) model. Namely, in [18] under Poisson stimulation the output ISI pdf and mean ISI are obtained for the BN without feedback and with threshold (Th) 2 , and the mean ISI for $\mathrm{Th}=3$. In $[8,29,30]$ a $\mathrm{BN}$ model with $\mathrm{Th}=2$ and with delayed feedback, either excitatory or inhibitory, stimulated with a Poisson stream is considered. For this case, the ISI pdf is found and also it is proven that the output ISI stream is non-Markov. In [13], any neuronal model from a defined class is considered. A delayed feedback is assumed excitatory, and stimulation is Poissonian. For 


\section{A. K. VIDYBIDA}

this case, it is proven that the output stream is nonMarkov. In this paper, a class of neuronal models with delayed Cl-type inhibitory feedback is considered.

The memory property in output ISI streams is often discussed in terms of correlation coefficient (CC), e.g. [42]. Unfortunately, the expressions obtained in this paper cannot be used for conclusions made in terms of CC. This is because all expressions, including $p\left(t_{1}, t_{0}\right)$ are obtained under restriction $(7)$, whereas in order to calculate CC one needs to know $p\left(t_{1}, t_{0}\right)$ for all $t_{0}>0$, $t_{1}>0$. Nevertheless, expressions derived in this paper under restriction (7) allow one to show that the Markov property is broken in the output ISI due to delayed feedback. Another reason why neuronal activity in a network is non-Markov is offered in [43].

In further work, it is expected to extend the exact ex- pressions obtained here to the full range of ISI values and to compare our findings with those obtained in terms of CC. This includes also a quantitative estimation of how much the statistics is non-Markov and to what extent it might be approximated by a Markov/renewal process. Also, a general renewal stochastic process can be considered as a stimulus instead of a Poisson one. The latter can be achieved if one finds an adequate expression for Eq. (13), which in its current form is valid for Poisson stimulation only.

Acknowledgements. This research was supported by theme grant of Department of Physics and Astronomy of NAS of Ukraine: "Dynamics of formation of spatially non-uniform structures in many-body systems", PK $0118 \mathrm{U} 003535$.

\section{APPENDIX}

Here we give a simple example of the proven property. Namely, we consider $p^{\text {inh }}\left(t_{2} \mid t_{1}, t_{0}\right)$ and show that $t_{0}$ dependence cannot be eliminated for a LIF model stimulated with a Poisson stream, see Fig. 2 below.

For the two values of $n=1,2$, Eq. (6), due to (7), (14), (18)-(20), (27), turns into the following two equations:

$$
\begin{aligned}
p^{\mathrm{inh}}\left(t_{1}, t_{0}\right) & =p^{0}\left(t_{1}\right) \int_{0}^{t_{0}} \mathbf{P}^{0}(s) p^{0}\left(t_{0}-s\right) g(s) d s+p^{0}\left(t_{0}\right) \int_{0}^{t_{1}} \mathbf{P}^{0}(s) p^{0}\left(t_{1}-s\right) g\left(s+t_{0}\right) d s \\
& +p^{0}\left(t_{1}\right) p^{0}\left(t_{0}\right) \int_{0}^{\Delta-t_{0}-t_{1}} f\left(s+t_{0}+t_{1}\right) d s \\
p^{\mathrm{inh}}\left(t_{2}, t_{1}, t_{0}\right) & =p^{\mathrm{inh}}\left(t_{2} \mid \Delta-t_{1}\right) p^{0}\left(t_{1}\right) \int_{0}^{t_{0}} \mathbf{P}^{0}(s) p^{0}\left(t_{0}-s\right) g(s) d s \\
& +p^{\mathrm{inh}}\left(t_{2} \mid \Delta\right) p^{0}\left(t_{0}\right) \int_{0}^{t_{1}} \mathbf{P}^{0}(s) p^{0}\left(t_{1}-s\right) g\left(s+t_{0}\right) d s \\
& +p^{0}\left(t_{1}\right) p^{0}\left(t_{0}\right) \int_{0}^{\Delta-t_{0}-t_{1}} p^{\mathrm{inh}}\left(t_{2} \mid s\right) f\left(s+t_{0}+t_{1}\right) d s,
\end{aligned}
$$

Now, let the neuronal model be the basic LIF model characterized with the firing threshold $V_{0}$, input impulse height $h$ and relaxation time $\tau$. Assume that

$$
0<h<V_{0}<2 h
$$

Assume also that the neuron is stimulated with a Poisson stream of intensity $\lambda$. For this case, it is proven in ( [19, Eqs. (14),(21)] that

$$
p^{0}(t)=\lambda^{2} t e^{-\lambda t}, \text { provided } t \in\left[0 ; T_{2}\right], \text { where } T_{2}=\tau \log \left(h /\left(V_{0}-h\right)\right) .
$$

Assume, for simplicity, that $\Delta<T_{2}$. This allows to obtain exact expressions for $\mathbf{P}^{0}(s)$ and $p^{\text {inh }}(t \mid s)$ :

$$
\begin{gathered}
\mathbf{P}^{0}(s)=e^{-\lambda s}(\lambda s+1), \\
p^{\mathrm{inh}}(t \mid s)=\lambda^{2} e^{-\lambda t}(t \chi(s-t)+\chi(t-s)(t-s)(\lambda s+1)) .
\end{gathered}
$$


If we put $\chi(0)=0.5$, then $\chi(-x)=1-\chi(x)$ and the last expression can be transformed as follows

$$
p^{\operatorname{inh}}(t \mid s)=\lambda^{2} e^{-\lambda t}(t+\chi(t-s) s(\lambda(t-s)-1)) .
$$

Under the assumptions of this Appendix, it appears that the kernel of integral equation (16) above is exactly the same as for the binding neuron model with excitatory feedback. The latter case has been studied in [30, Eqs. (14)(16)], where the unique solution to Eq. (16) is found. The unknown quantities from Eq. (17) above under assumptions of this Appendix can be taken from [30]:

$$
g(s)=a \lambda\left(1-e^{-2 \lambda(\Delta-s)}\right) / 2, \quad a=4 e^{2 \lambda \Delta} /\left((2 \lambda \Delta+3) e^{2 \lambda \Delta}+1\right) .
$$

After substituting (32)-(35) into (29) one obtains

$$
\begin{aligned}
& p^{\operatorname{inh}}\left(t_{1}, t_{0}\right)=\frac{\lambda^{4} e^{-\lambda\left(t_{0}+t_{1}\right)} t_{0} t_{1}}{6\left((2 \lambda \Delta+3) e^{2 \lambda \Delta}+1\right)} \\
& \times\left(2 \lambda e^{2 \lambda \Delta}\left(\lambda\left(t_{0}^{2}+t_{1}^{2}\right)+3\left(2 \Delta-t_{0}-t_{1}\right)\right)+3\left(e^{2 \lambda\left(t_{1}+t_{0}\right)}+6 e^{2 \lambda \Delta}+1\right)\right) .
\end{aligned}
$$

Notice, that $p^{\mathrm{inh}}\left(t_{1}, t_{0}\right)$ is strictly positive for strictly positive $t_{1}, t_{0}$. This allows one to use it safely as denominator in the definition of conditional probability (5) above. Also, as it may be observed from $(36), p^{\text {inh }}\left(t_{1}, t_{0}\right)$ is continuous and bounded. This means that $p^{\text {inh }}\left(t_{2} \mid t_{1}, t_{0}\right)$, as it is defined in (5), will preserve any discontinuity which may appear in $p^{\text {inh }}\left(t_{2}, t_{1}, t_{0}\right)$, which is the numerator in (5) for $n=1$.

Consider now Eq.(30) for $p^{\text {inh }}\left(t_{2}, t_{1}, t_{0}\right)$. After partial simplifications, it turns into the following:

$$
\begin{gathered}
p^{\text {inh }}\left(t_{2}, t_{1}, t_{0}\right)=p^{\text {inh }}\left(t_{2} \mid \Delta-t_{1}\right) \frac{\lambda^{4} t_{0} t_{1} e^{-\lambda\left(t_{0}+t_{1}\right)}}{6\left((2 \lambda \Delta+3) e^{2 \lambda \Delta}+1\right)}\left(3\left(1-e^{2 \lambda t_{0}}\right)+2 \lambda t_{0} e^{2 \lambda \Delta}\left(\lambda t_{0}+3\right)\right) \\
+p^{\text {inh }}\left(t_{2} \mid \Delta\right) \frac{\lambda^{4} t_{0} t_{1} e^{-\lambda\left(t_{0}+t_{1}\right)}}{6\left((2 \lambda \Delta+3) e^{2 \lambda \Delta}+1\right)}\left(3 e^{2 \lambda t_{0}}\left(1-e^{2 \lambda t_{1}}\right)+2 \lambda t_{1} e^{2 \lambda \Delta}\left(\lambda t_{1}+3\right)\right) \\
+\lambda^{2} t_{2} e^{-\lambda t_{2}} p^{0}\left(t_{1}\right) p^{0}\left(t_{0}\right) \chi\left(\Delta-t_{0}-t_{1}-t_{2}\right) \int_{t_{2}}^{\Delta-t_{0}-t_{1}} g\left(s+t_{0}+t_{1}\right) d s \\
+\lambda^{2} e^{-\lambda t_{2}} p^{0}\left(t_{1}\right) p^{0}\left(t_{0}\right) \int_{0}^{\min \left(t_{2}, \Delta-t_{0}-t_{1}\right)}\left(t_{2}-s\right)(\lambda s+1) g\left(s+t_{0}+t_{1}\right) d s \\
+a p^{0}\left(t_{1}\right) p^{0}\left(t_{0}\right) p^{\mathrm{inh}}\left(t_{2} \mid \Delta-t_{0}-t_{1}\right) .
\end{gathered}
$$

The summands (a) and (b) in Eq. (37) correspond to the first and second term of the right-hand side in Eq.(30), respectivly. The remaining three correspond to the third term of the right-hand side in Eq. (30). It is easily seen that both (a) and (b) are continuous with respect to $t_{0}$. Term (d) is also continuous in $t_{0}$, because function min $(x, y)$ is continuous on $x$ and $y$. Term (c) is also continuous because

$$
\lim _{\Delta-t_{0}-t_{1}-t_{2} \rightarrow 0} \chi\left(\Delta-t_{0}-t_{1}-t_{2}\right) \int_{t_{2}}^{\Delta-t_{0}-t_{1}} g\left(s+t_{0}+t_{1}\right) d s=0 .
$$

Consider the final term in Eq. (37). For the sake of clarity, we omit the factor $a p^{0}\left(t_{1}\right) p^{0}\left(t_{0}\right)$, having in mind that it is continuous and strictly positive for $t_{0}>0, t_{1}>0$. The remaining expression is as follows

$$
\lambda^{2} e^{-\lambda t_{2}}\left(t_{2}-\chi\left(t_{2}+t_{1}+t_{0}-\Delta\right)\left(\Delta-t_{0}-t_{1}\right)\left(\lambda\left(\Delta-t_{0}-t_{1}-t_{2}\right)+1\right)\right) .
$$




\section{A. K. VIDYBIDA}
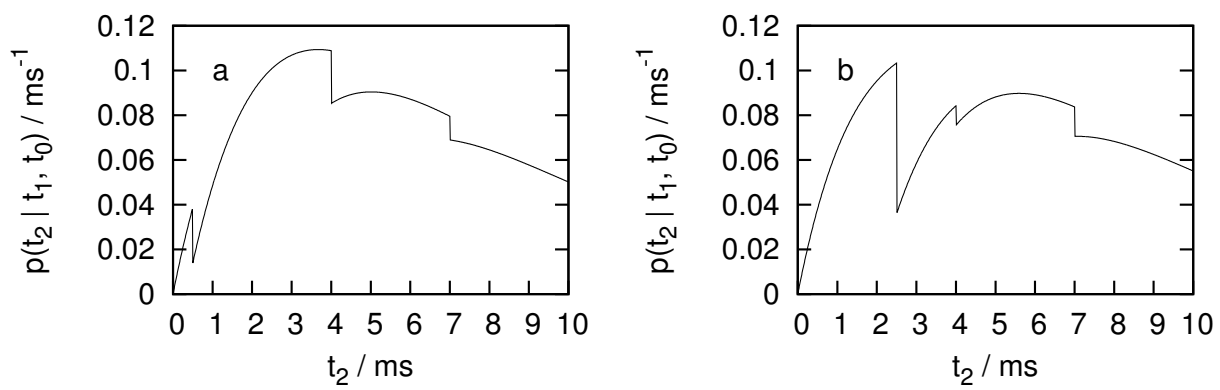

Fig. 2. Different values of $p^{\mathrm{inh}}\left(t_{2} \mid t_{1}, t_{0}\right)$ for different $t_{0}$. Here $\Delta=7 \mathrm{~ms}, \lambda=0.3 \mathrm{~ms}^{-1}, t_{1}=3 \mathrm{~ms}$ both for (a) and (b). Time $t_{0}=3.5 \mathrm{~ms}$ for (a) and $t_{0}=1.5 \mathrm{~ms}$ for (b). The curves are calculated based on Eqs. (34)-(37).

This expression, if considered as a function of $t_{0}, t_{1}, t_{2}$, has a step-like discontinuity along the hyperplane

$$
t_{2}+t_{1}+t_{0}=\Delta .
$$

Indeed, if $t_{2}+t_{1}+t_{0}<\Delta$, then (38) turns into $\lambda^{2} e^{-\lambda t_{2}} t_{2}$. Otherwise, if $t_{2}+t_{1}+t_{0}>\Delta$, then (38) turns into $\lambda^{2} e^{-\lambda t_{2}}\left(t_{2}-\left(\Delta-t_{0}-t_{1}\right)\left(\lambda\left(\Delta-t_{0}-t_{1}-t_{2}\right)+1\right)\right)$. The difference between the two expressions is as follows

$$
\lambda^{2} e^{-\lambda t_{2}}\left(\Delta-t_{0}-t_{1}\right)\left(\lambda\left(\Delta-t_{0}-t_{1}-t_{2}\right)+1\right) .
$$

This difference vanishes along the hyperplane $t_{2}+t_{1}+t_{0}=\Delta+\frac{1}{\lambda}$ only. (Due to (7), we do not consider the case $\left.t_{0}+t_{1}=\Delta\right)$. Comparing the last equation with (39), we see that the jump (40) is strictly positive along the hyperplane (39). The same is valid for the last term in (37). Taking into account that the other four terms in (37) are continuous in $t_{0}$, and what is said after Eq. (36), we conclude that $p^{\text {inh }}\left(t_{2} \mid t_{1}, t_{0}\right)$ has a nonzero jump along the hyperplane (39). For a fixed $t_{1}, t_{2}$ and infinitesimally small $\epsilon>0$ consider two different values of $t_{0}: t_{0}^{ \pm}=\Delta-t_{1}-t_{2} \pm \epsilon$. It is clear from the above that when $t_{0}$ value obtains an infinitesimally small change from $t_{0}^{+}$to $t_{0}^{-}$, function $p^{\operatorname{inh}}\left(t_{2} \mid t_{1}, t_{0}\right)$ gets a finite change due to the jump (40), which means that $t_{0}$-dependence in $p^{\operatorname{inh}}\left(t_{2} \mid t_{1}, t_{0}\right)$ is indeed present (at least due to the discovered jump discontinuity) and cannot be eliminated. This is illustrated in Fig. 2.

[1] R. B. Stein, Biophys. J. 7, 37 (1967).

[2] H. L. Bryant, J. P. Segundo, J. Physiol. 260, 279 (1976).

[3] R. M. Capocelli, L. M. Ricciardi, Kybernetik 8, 214 (1971).

[4] S. Ghosh-Dastidar, H. Adeli, Int. J. Neural. Syst. 19, 295 (2009).

[5] L. Sacerdote, M. T. Giraudo, in Stochastic Biomathematical Models, Lecture Notes in Mathematics 2058, edited by M. Bachar, J. J. Batzel, S. Ditlevsen (Springer-Verlag, 2013), p. 99.

[6] R. Sarpeshkar, in McGraw-Hill Yearbook of Science 85 Technology 2009 (New York, McGraw-Hill, 2009), p. 250.

[7] D. Bruderle et al., Biol. Cybern. 104, 263 (2011).

[8] K. G. Kravchuk, A. K. Vidybida, BioSystems 112, 233 (2013).

[9] A. K. Vidybida, in Encyclopedia of information science and technology, edited by M. Khosrow-Pour (IGI Global, 2014), p. 1123.

[10] B. Lindner, in Stochastic Methods in Neuroscience, edited by C. Laing, G. J. Lord (Oxford University Press, 2010), p. 1.

[11] J. L. Doob, Stochastic processes (New York, Wiley, 1953).

[12] N. G. van Kampen, Braz. J. Phys. 28, 90 (1998).

[13] A. K. Vidybida, J. Stat. Phys. 160, 1507 (2015).

[14] A. N. Burkitt, Biol. Cybern. 95, 1 (2006).

[15] M. J. Chacron, K. Pakdaman, A. Longtin, Neural Comput. 15, 253 (2003).
[16] R. Jolivet, T. J. Lewis, W. Gerstner, J. Neurophysiol. 92, 959 (2004).

[17] R. Jolivet, A. Rauch, H. Luscher, W. Gerstner, J. Comput. Neurosci. 21, 35 (2006).

[18] O. K. Vidybida, Ukr. Math. J. 59, 1819 (2007).

[19] A. K. Vidybida, Rap. Acad. Sci. Ukr., No. 12, 18 (2014).

[20] J. M. Bekkers, Curr. Biol. 8, R52 (1998).

[21] A. Bacci, J. R. Huguenard, D. A. Prince, J. Neurosci. 23, 859 (2003).

[22] A. Bacci, J. R. Huguenard, D. A. Prince, Nature, 431, $312(2004)$.

[23] T. C. Smith, C. E. Jahr, Nat. Neurosci. 5, 760 (2002).

[24] L. S. Benardo, J. Physiology 476.2, 203 (1994).

[25] J. F. Storm, Biophys. J. 53, 148a (1988).

[26] J. Storm, Prog. Brain Res. 83, 161(1990).

[27] J. L. Rossello, V. Canals, A. Morro, A. Oliver, Int. J. Neural Syst. 22, 1250014 (2012).

[28] R. Wang, G. Cohen, K. M. Stiefel, T. J. Hamilton, J. Tapson, A. van Schaik, Front. Neurosci. 7, 1 (2013).

[29] A. K. Vidybida, K. G. Kravchuk, BioSystems 112, 224 (2013).

[30] A. K. Vidybida, in 14th International Congress of Cybernetics and Systems of WOSC, Wroctaw, Poland, September 9-12, 2008, edited by J. Jozefczyk, W. Thomas, M. Turowska (Oficyna Wydawnicza Politechniki Wrocławskiej, 2008), p. 292.

[31] B. B. Averbeck, Neuron 62, 310 (2009). 
[32] S. B. Lowen, M. C. Teich, J. Acoust. Soc. Am. 92, 803 (1992).

[33] S. Shinomoto, Y. Sakai, S. Funahashi, Neural Comput. 11, 935 (1999).

[34] R. Ratnam, M. E. Nelson, J. Neurosci. 20, 6672 (2000).

[35] M. P. Nawrot et al., Neurocomputing 70, 1717 (2007).

[36] G. Maimon,d J. A. Assad, Neuron 62, 426 (2009).

[37] J. P. Rospars, P. Lánský, Biol. Cybern. 69, 283 (1993).

[38] P. Lánský, R. Rodriguez, Physica D 132, 267 (1999).
[39] E. Benedetto, L. Sacerdote, Biol. Cybern. 107, 95 (2013)

[40] R. E. Kass, V. Ventura, E. N. Brown, J. Neurophys. 94 8 (2005).

[41] O. Avila-Akerberg, M. J. Chacron, Exp. Brain Res. 210, 353 (2011).

[42] F. Farkhooi, E. Muller, M.P. Nawrot, Phys. Rev. E 83, 050905 (2011)

[43] B. Cessac, J. Math. Biol. 62, 863 (2011).

\title{
СТАТИСТИКА ВИХІДНОЇ АКТИВНОСТІ ГАЛЬМІВНОГО НЕЙРОНА ЗІ ШВИДКИМ, СІ-ПОДІБНИМ ГАЛЬМУВАННЯМ І З ЗАТРИМАНИМ ЗВОРОТНІМ ЗВ'ЯЗКОМ - НЕМАРКІВСЬКА
}

\author{
О. К. Відибіда \\ Інститут теоретичної фізики ім. М. М. Боголюбова \\ вул. Метрологічна, 14-б, Київ, 03680, Україна \\ e-mail:vidybida@bitp.kiev.ua
}

\begin{abstract}
Для класу моделей імпульсних нейронів гальмівного типу зі швидким, Cl-подібним гальмуванням і з затриманим зворотним зв'язком, стимульованих пуассонівським випадковим процесом збуджувальних імпульсів доведено, що вихідний потік міжімпульсних інтервалів не можливо подати як процес Маркова будь-якого порядку.
\end{abstract}

